\title{
Characterization of LC3 and p62 on Rat Prostate Lobe in Benign Prostate Hyperplasia Animal Model
}

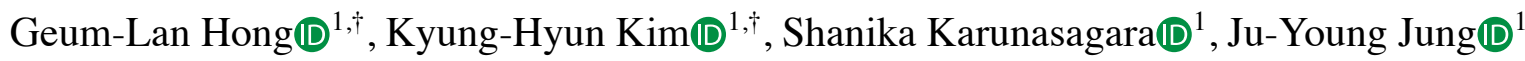 \\ ${ }^{1}$ Department of Veterinary Medicine \& Institute of Veterinary Science, Chungnam National University
}

\begin{abstract}
Benign prostatic hyperplasia (BPH) is disease characterized by abnormal prostate cell proliferation. Rat models of testosterone propionate (TP)-induced BPH are the most popular experimental models for studying BPH. In rats, the prostate is located below the base of the urinary bladder and comprises four distinct lobes ventral, lateral, dorsal, and anterior. Autophagy associated with cellular homeostasis has been studied in relation to BPH. Microtubule-associated proteins 1A/1B-light chain 3 (LC3B) and sequestosome 1 (p62) are key markers of autophagy flux. However, the expression and localization of LC3B and p62 have not been elucidated in rats with testosterone-induced BPH. This study investigated the expression and specific localization of the two autophagy markers mentioned. Fifteen Sprague-Dawley rats were classified into three groups: normal control (N.C.), BPH (TP $5 \mathrm{mg} / \mathrm{kg}$ ), and Fina (TP + finasteride $10 \mathrm{mg} / \mathrm{kg}$ ). To evaluate the expression of the autophagy markers in $\mathrm{BPH}$, hematoxylin and eosin staining and immunohistochemistry were performed for LC3B and p62. Both LC3B expression and p62 expression were higher in the anterior lobe than other areas. In addition, there was no significant difference in the dorsal lobe LC3B expression among the N.C., BPH, and Fina groups. In the lateral lobe, LC3B expression was decreased in the BPH group and increased in the Fina group. p62 expression in the BPH and Fina groups increased compared to that in the N.C. group. In the ventral lobe of the prostate, LC3B expression was lower in the BPH group, whereas it was higher in the Fina group. On the other hand, p62 expression increased in the BPH group, whereas it was lower in the Fina group similar to those observed in the N.C. group. Autophagy was suppressed in the BPH group, whereas it was induced in the ventral lobe in the Fina group. Based on our finding, we suggest that autophagy is a critical process in BPH. In particular, the ventral lobe of the rat prostate could be a potential target site for evaluating the therapeutic effects of BPH treatment in animal models.
\end{abstract}

Keywords : Localization, Autophagy, LC3, p62, Benign prostatic hyperplasia

This work was supported by research fund of Chungnam National University. 'These authors contributed equally to this work

The author(s) agree to abide by the good publication practice guideline for medical journals.

The author(s) declare that there are no conflicts of interest.

Received: November 19, 2020; Revised: December 22, 2020;

Accepted: December 22, 2020

Correspondence to: Ju-Young Jung (College of Veterinary Medicine, Chungnam National University, 220 Gung-dong, Yusung-gu, Daejeon 34134, Republic of Korea)

E-mail: jyjung@cnu.ac.kr

\section{INTRODUCTION}

Benign prostatic hyperplasia (BPH) is an age-related proliferative disease of the prostate prevalent among elderly male. Histopathologically, BPH is characterized by increased number of epithelial and stromal cells in the periurethral area of the prostate gland [1]. Clinically, BPH is characterized by benign enlargement of the transitional zone 
in the prostate, and is accompanied by lower urinary tract symptoms, which affect the quality of life in patients [2].

Rat prostate responds to hormonal treatment. Scolnik et al. [3] reported that Wistar and Sprague-Dawley (SD) strains were most susceptible to BPH induction following treatment with exogenous testosterone. The rat prostate comprises four distinct lobes - ventral, lateral, dorsal, and anterior (coagulating gland) - with different morphological characteristics. These lobes are classified according to their position relative to the urinary bladder [4-7]. The lateral prostate is the site of prostatitis related to aging and hormones expressions $[8,9]$. The ventral prostate is located on the ventral side of the urethra, immediately below the bladder, and contains approximately half of the total prostatic tissue [10]. The lateral and ventral lobes of the prostate are the sites of androgenic action and $\mathrm{BPH}[11,12]$.

Autophagy is an evolutionarily conserved process that enables cells to maintain cellular homeostasis during starvation and other stressful conditions [13]. Therefore, autophagy can be regulated in response to various diseases including BPH $[14,15]$. Autophagy is induced by the formation of a characteristic double-membraned vesicle known as the autophagosome. Autophagosome formation is regulated by microtubule-associated protein 1 light chain 3B (LC3B) and ubiquitin-binding protein SQSTM1/p62 (p62). Since p62 associates with LC3B present on autophagosomal membranes and is subsequently degraded by the autophagylysosomal system [16-18], LC3B and p62 are key markers for monitoring autophagy $[19,20]$. Dysregulation of autophagy is related with the pathogenesis of carcinoma, neurodegenerative disease, inflammatory diseases, as well as the prostate disease [15].

Several recent studies have shown that autophagy and autophagy-related proteins are associated with BPH development [21-23]. In a clinical study, autophagy was induced in BPH tissues, especially in prostate stromal cells, after a long duration of 5-ARI treatment [24]. Deactivation of autophagy is associated with prostatic cells of patients with lower urinary tract symptoms (LUTS) and BPH [25]. In rat $\mathrm{BPH}$, autophagy is decreased in prostate gland, which is demonstrated through the results of the suppression of autophagic flux [26]. In another study, LC3B also showed that expression decreased in testosterone-induced prostate hyperplasia in rats [27,28].

In human prostate, transitional and peripheral zone is implicated in the main site for BPH and also been focused on apoptosis and shrinkage of the glandular epithelial compartment by $5 \alpha$-reductase inhibitor [1]. However, it is yet to be known about the site for action of prostate lobe in rat corresponding to transition and peripheral zone in human. Therefore, identification of the site of autophagic localization and determination of the mechanism underlying the autophagy-mediated regulation of specific targets in $\mathrm{BPH}$ are major issues to be addressed. This study examined the specific localization of LC3 and p62 and compared their levels in each lobe of the prostate in a rat model of BPH using western blotting and immunohistochemistry (IHC).

\section{MATERIALS AND METHODS}

\section{Experimental animals}

The experimental protocols were approved by the International Animal Ethics Committee of Chungnam National University (CNU-01108). Six-week-old, male SD rats were purchased from Orient Bio (Gyeonggi-do, Korea) and were housed in a specific-pathogen-free animal facility under controlled conditions of temperature, humidity, and photoperiod $\left(22 \pm 2^{\circ} \mathrm{C}, 55 \% \pm 5 \%\right.$, and 12-h light/dark cycle, respectively) for 1 week prior to the experiment. All animals were fed a standard chow diet and provided ad libitum access to water.

\section{Experimental design}

The SD rats were randomly classified into three groups ( $\mathrm{n}=$ five per group). To induce $\mathrm{BPH}$, all rats except the rats in the normal control group, rats were subcutaneously (S.C.) injected with testosterone propionate (TP) $(5 \mathrm{mg} / \mathrm{kg}$ dissolved in corn oil, Tokyo Chemicals Ins. Co., Tokyo, Japan) daily for 4 weeks. To minimize stress during the treatment, the S.C. injection was administered in the loose skin on the dorsal surface of the rat, and the site of injection was varied to reduce local reactions in the skin. Finasteride, a drug widely used to treat BPH that acts by inhibiting $5 \alpha$-reductase activity [29], was administered orally for four weeks. The treatment groups were as follows: normal control (N.C.) group (Corn oil $1 \mathrm{~mL} / \mathrm{kg}$, S.C. + PBS, per oral), BPH group (TP $5 \mathrm{mg} / \mathrm{kg}$, S.C. + PBS, per oral), and Fina group (TP $5 \mathrm{mg} /$ $\mathrm{kg}$, S.C. + finasteride $10 \mathrm{mg} / \mathrm{kg}$, per oral). At the end of the experimental period, the rats were fasted overnight and euthanized by inhalation of $\mathrm{CO}_{2}$ gas for $5 \mathrm{~min}$ in a euthana- 
sia apparatus. The dissected prostate glands were weighed and stored at $-80^{\circ} \mathrm{C}$ in liquid nitrogen for further analysis. The body weight of each rat was measured at the beginning and end of the experimental period.

\section{Histological study}

Paraffin-embedded prostate tissues were cut into 5- $\mu \mathrm{m}$ sections. After deparaffinization and dehydration, the sections were subjected to hematoxylin and eosin (H\&E) staining and observed using a light microscope (Nikon eclipse $80 \mathrm{i}$, Nikon Corporation, Tokyo, Japan) at $400 \times$ magnification. Images were acquired from ten randomly selected fields, and epithelial thickness was measured using Image J software (Image J v1.46a; NIH, USA).

\section{Western blot analysis}

The frozen prostate tissue samples were homogenized and lysed using a radioimmunoprecipitation assay buffer (50 $\mathrm{mM}$ Tris-HCl, $\mathrm{pH}$ 8.0, $150 \mathrm{mM}$ sodium chloride, $1 \%$ NP-40, $0.5 \%$ sodium dodecyl sulfate (SDS), and a protease inhibitor cocktail). The homogenate was centrifuged at 12,000 rpm for $20 \mathrm{~min}$ at $4^{\circ} \mathrm{C}$, and the supernatant was used for western blotting analysis. Protein samples were separated by electrophoresis on $8 \% \sim 15 \%$ SDS polyacrylamide gels, and the separated proteins were transferred onto a polyvinylidene fluoride membrane using a semi-dry transfer system (BioRad, Hercules, CA, USA). The membrane was blocked with $5 \%$ dry milk in PBS-T and treated using anti-LC3B (1: 1000; Sigma-Aldrich, MO, USA) and anti-p62 (1:1000; Cell Signaling, Danvers, MA, USA) antibodies as primary antibodies. Subsequently, the blots were incubated for $1 \mathrm{~h}$ with horseradish peroxidase-conjugated goat anti-rabbit (1:5000, Ab Frontier, Seoul, Korea) IgG used as the secondary antibody to identify each protein. The proteins were visualized using an enhanced chemiluminescence detection kit (Amersham Pharmacia Biotech, Buckinghamshire, UK) and quantified using Image Lab Software (Bio-Rad).

\section{IHC analysis}

IHC analysis was performed using the Vectastain Elite ABC kit (Vector Laboratories, Burlingame, USA) according to the manufacturer's instructions. After deparaffinization and dehydration, the sections were treated with $3 \%$ quenched endogenous $\mathrm{H}_{2} \mathrm{O}_{2}$ (in methanol) and $0.5 \%$ Triton X-100 solution for $30 \mathrm{~min}$ at room temperature $\left(25^{\circ} \mathrm{C}\right)$. Next, the non-specific binding sites were blocked by treating with normal goat serum (diluted in $1: 10$ ratio with $\mathrm{PBS}$ ), and the sections were treated overnight at $4^{\circ} \mathrm{C}$ with anti-LC3B (1 : 100; Sigma-Aldrich) and anti-p62 (1:100; Cell Signaling) antibodies (primary antibodies), followed by treatment with a goat anti-rabbit secondary antibody $(1: 200, \mathrm{Ab}$ Frontier). For developing the sections, a diaminobenzidine peroxidase substrate kit (Vector Laboratories) was used. The LC3B- and p62-positive areas in the stained sections were visualized as brown-colored regions under a light microscope (Nikon eclipse $80 \mathrm{i}$ ) at $400 \times$ magnification at ten different locations. The IHC test gives a score of 0 tor $3+$ that measures the intensity of LC3B- and p62-positive cells in the prostate tissue samples; one or little stained cells $(0)$; weak, partial stained cells (1); intermediate, complete stained cells (2); strong, complete membrane stained cells (3).

\section{Statistical analysis}

All experiments were conducted in a double-blinded manner. Results were randomly selected and expressed as mean \pm standard deviation. Statistical analysis was performed using one-way analysis of variance, followed by post-hoc analysis using Holm-Sidak's multiple comparison

Table 1. The body weights and prostate weights in testosterone-induced benign prostate hyperplasia in rat

\begin{tabular}{lccrr}
\hline & \multicolumn{2}{c}{ Body weight $(\mathrm{g})$} & \multicolumn{2}{c}{ Prostate weight $(\mathrm{g})$} \\
& Initial & Final & Absolute & Relative \\
\hline N.C. & $224.1 \pm 2.27$ & $363.3 \pm 17.5$ & $0.703 \pm 0.057$ & $0.193 \pm 0.01$ \\
BPH & $224.4 \pm 1.91$ & $331.5 \pm 9.4$ & $1.296 \pm 0.035^{\# \# \#}$ & $0.391 \pm 0.05^{\# \# \#}$ \\
Fina & $224.6 \pm 1.93$ & $338.4 \pm 8.5$ & $1.006 \pm 0.092^{*}$ & $0.297 \pm 0.03^{* *}$ \\
\hline
\end{tabular}

Testosterone-induced BPH rat models were administered finasteride $(10 \mathrm{mg} / \mathrm{kg}$ P.O.) for 28 days. The values are expressed as mean \pm standard deviation,

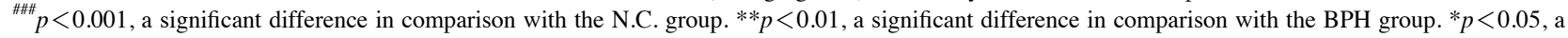
significant difference compared to the BPH group. 
A
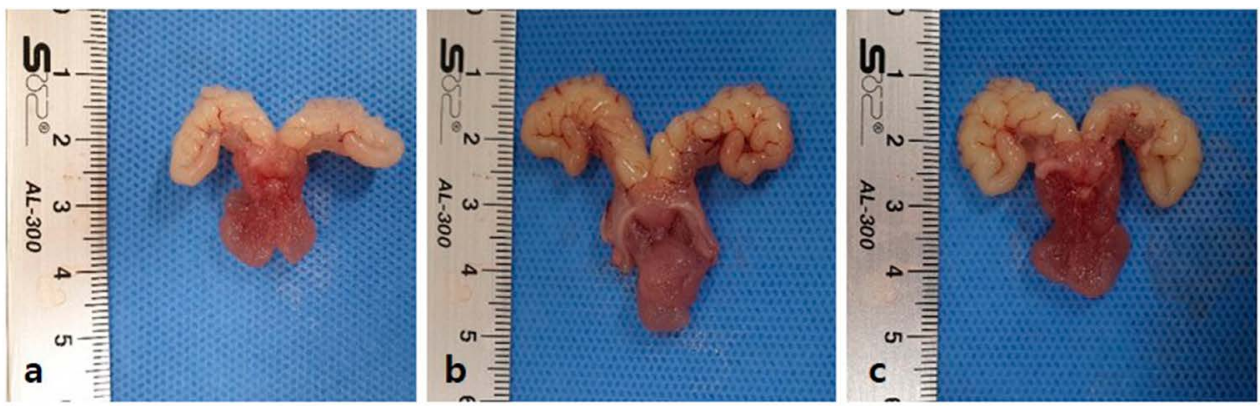

B
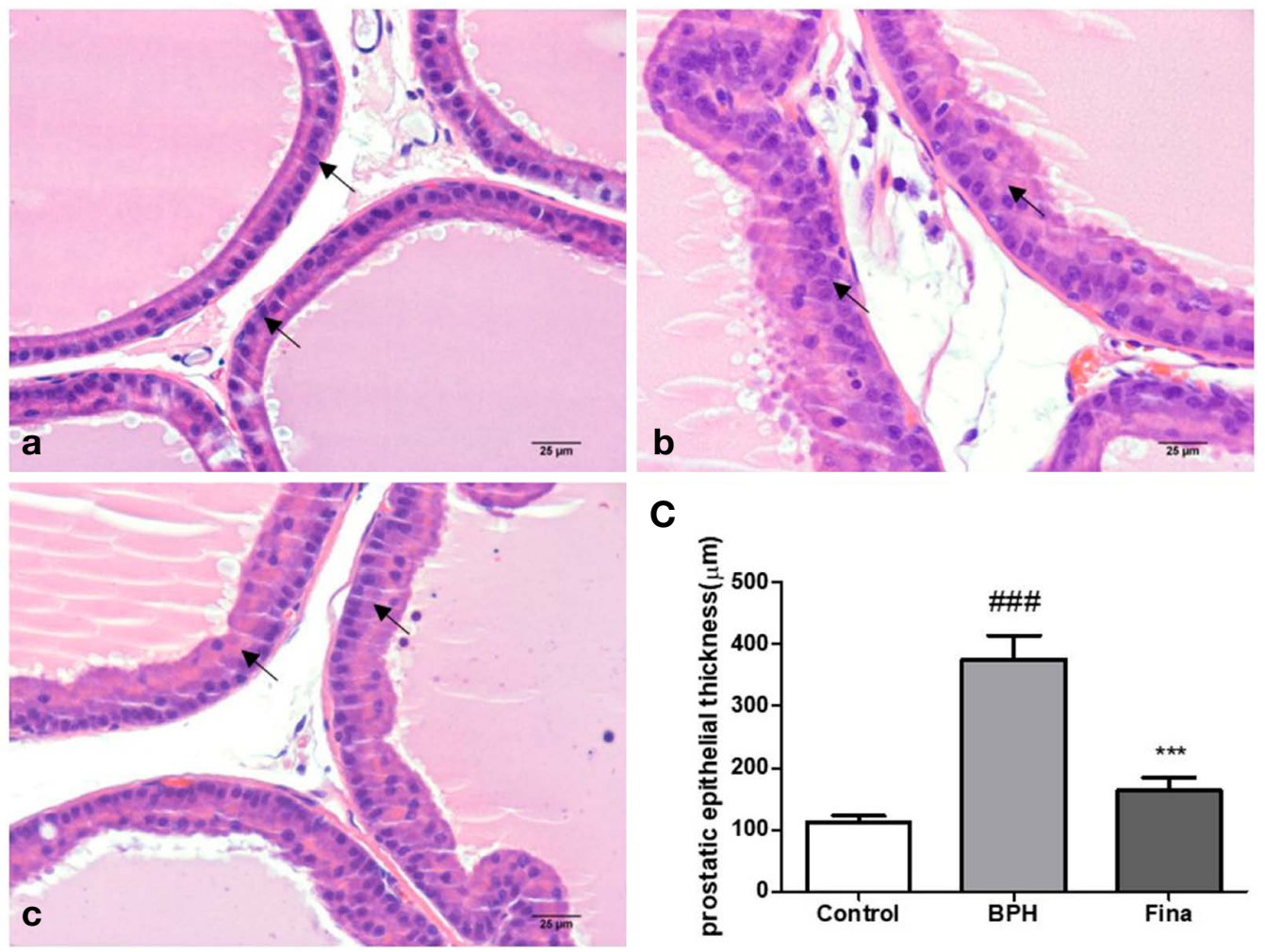

C

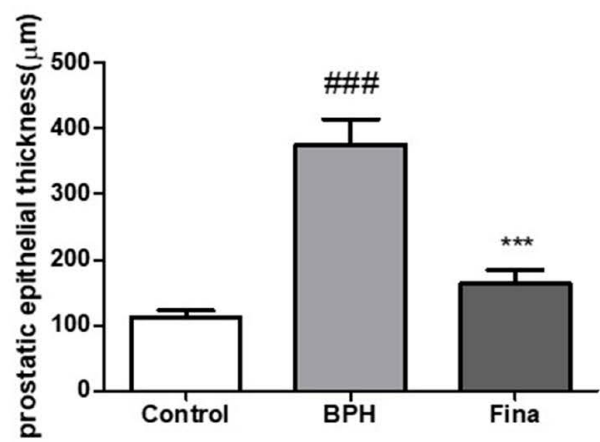

Fig. 1. Histopathology of prostate tissue sections determined by hematoxylin and eosin (H\&E) staining. Scale bar $=25 \mu \mathrm{m}$. Rats with testosterone-induced BPH were administered with finasteride (10 mg/kg P.O.) for 28 days. A. Prostate; (a) N.C.; PBS (b) BPH group; TP (c) Fina group; TP + Finasteride (10 mg/kg P.O.). B. H\&E. C. The thickness of prostatic epithelial cells (arrow) in the BPH group was increased. In contrast, in finasteride group, it was significantly reduced. Values are expressed as mean \pm standard deviation, ${ }^{\# \#} p<0.001$, a significant difference in comparison with the N.C. group. ${ }^{* *} p<0.001$, a significant difference in comparison with the BPH group.

test. Results with $p$ values $<0.05$ were considered statistically significant.

\section{RESULTS}

\section{Effect of testosterone on body weight and prostate weight}

We analyzed the body and prostate weights of rats. As shown in Table 1, the increase in the body weights of rats with $\mathrm{BPH}$ was less than that in the normal group, with no significant difference. However, the absolute and relative prostate weights/body weights increased significantly $(p<0.001)$ and were twice as high as those in the N.C. group. Finasteride treatment significantly decreased $(p<0.01)$ the relative prostate weights.

\section{Histopathological changes in rats with testosterone-induced $\mathrm{BPH}$}

BPH was diagnosed histopathologically by $\mathrm{H} \& \mathrm{E}$ staining. 
The morphological changes in the ventral lobe of the prostate are shown in Figure 1. The prostate size was greater in the BPH group that in the N.C. group. However, the prostate size reduced in the Fina group (Fig. 1A). No histological changes were observed in the N.C. group. Testosterone treatment led to thickening of the ventral lobe of the prostate (Fig. 1B). In particular, the epithelial thickness in the $\mathrm{BPH}$ group was 3.3-fold greater than that in the N.C. group $(p<0.001)$. Compared to the BPH group, the finasteridetreated group showed significantly reduced epithelial thickness $(p<0.001)$ (Fig. 1).

\section{LC3B and p62 expression in rats with testosterone-induced BPH}

As shown in Figure 2, western blotting was performed to evaluate the expression of the autophagy-related proteins LC3B and p62. LC3B expression was evaluated in the N.C. group. Testosterone treatment suppressed the LC3B expression compared to that in the N.C. group $(p<0.01)$. However, LC3B expression was higher in the Fina group than in the BPH group $(p<0.01)$. Rats with testosterone-induced
BPH showed increased p62 expression compared to N.C. rats $(p<0.001)$. However, finasteride treatment significantly suppressed $(p<0.001)$ p62 expression. These results suggest that finasteride treatment induced autophagy during $\mathrm{BPH}$ progression.

\section{LC3B and p62 IHC in the prostate lobe of rats with testosterone-induced BPH}

Based on the results of LC3B and p62 expression in western blotting, we analyzed the specific expression and localization of LC3B and p62 in rat prostate tissue using immunohistochemistry. As shown in Figure 3, LC3B expression was primarily detected in the epithelial cells of the prostate gland. In particular, LC3B expression was detected in the cytoplasm of epithelial cells. LC3B was expressed in all lobes of the prostate in N.C. group, particularly in the lateral and anterior lobes. Meanwhile, LC3B expression decreased in the BPH group, whereas it increased in the Fina group. In the BPH group, LC3B-positive cells were barely detected in the lateral and ventral lobes.

However, LC3B expression in the anterior, lateral, and
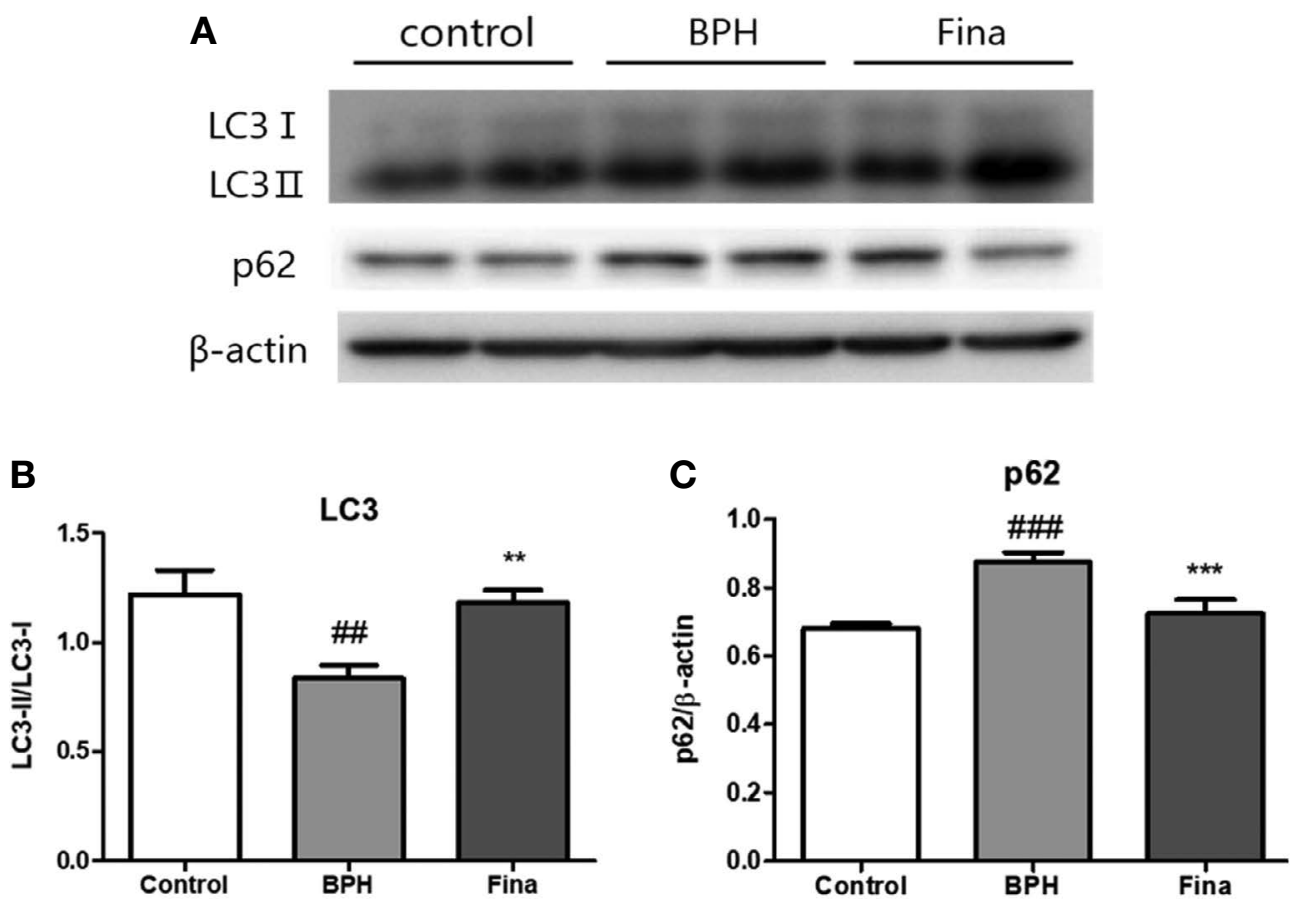

Fig. 2. Western blot analysis of LC3 and p62 using prostate tissues from rats with testosterone-induced BPH. Rats with testosterone-induced BPH were administered with finasteride $(10 \mathrm{mg} / \mathrm{kg}$ P.O.) for 28 days. A. Western blot analysis of LC3, p62 and $\beta$-actin at four weeks after testosterone administration. B. Graphical representation of the ratio of LC3B and p62 to $\beta$-actin. N.C.; PBS, BPH group; TP, Fina group; TP + Finasteride (10 mg/kg P.O.). Values are expressed as mean \pm standard deviation, ${ }^{\# \#} p<0.001$ and ${ }^{\# \#} p<0.01$, a significant difference in comparison with the N.C. group. ${ }^{* *} p<0.001$ and $* * p<0.01$, a significant difference in comparison with the BPH group. 
A

AL

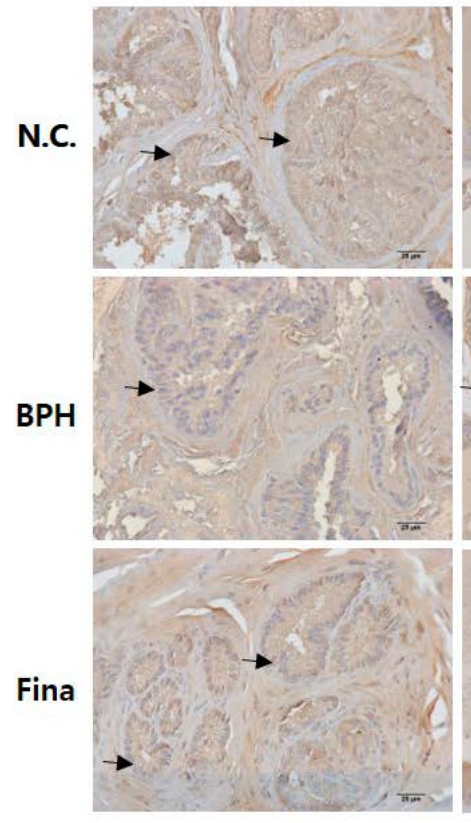

DL
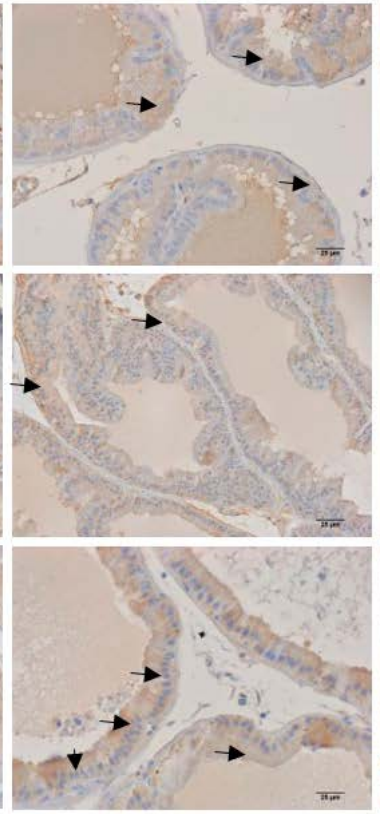

LL
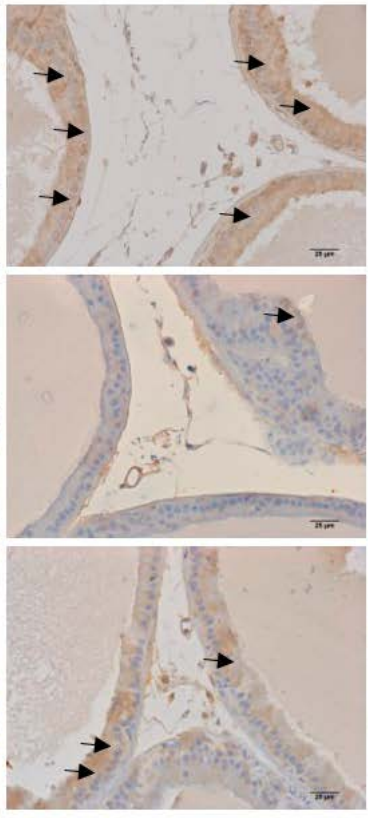

LC3

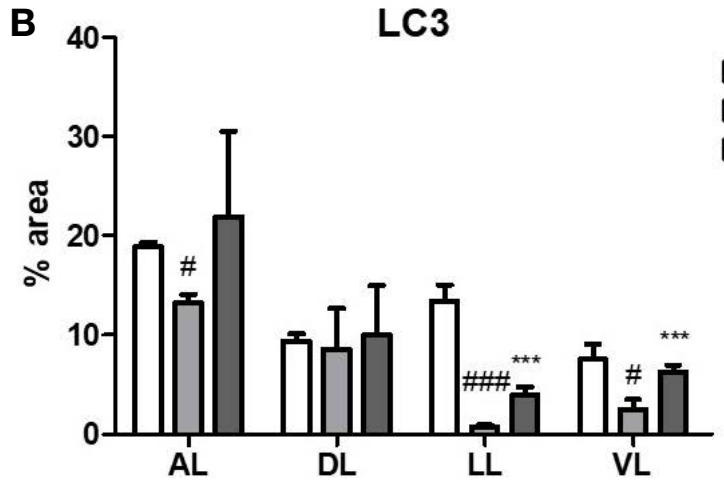

B
VL

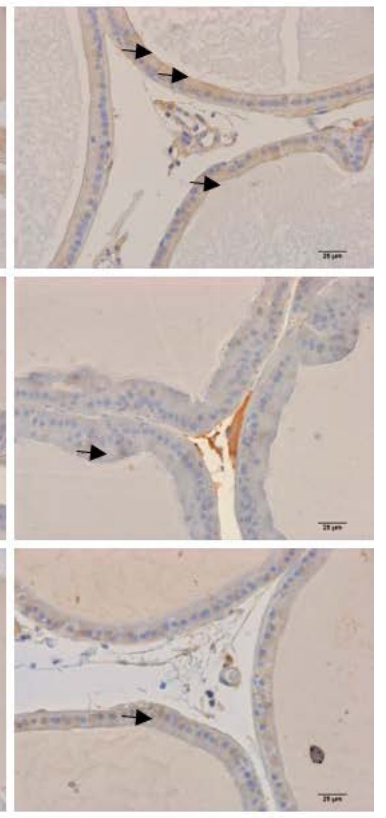

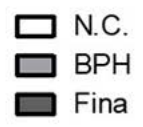

Fig. 3. Immunohistochemistry of LC3B in a rat model of testosterone-induced BPH. Scale bar $=25 \mu \mathrm{m}$. Rats with testosterone-induced BPH were administered with finasteride (10 mg/kg P.O.) for 28 days. A. Expression of LC3B (arrow) and examined by light microscopy with focus on AL, DL, LL, and VL. The\% area of positive LC3B (B) was expressed as mean \pm standard deviation, AL (anterior lobe), DL (dorsal lobe), LL (lateral lobe), and VL (ventral lobe), ${ }^{\# \#} p<0.001,{ }^{\#} p<0.05$, a significant difference in comparison with the N.C. group. $* * * p<0.001, * * p<0.01$, and $* p<0.05$, significant difference in comparison with the BPH group.

ventral lobes of the prostate increased in the Fina group compared to that in the BPH group. Surprisingly, there was no significant difference in LC3B expression in the dorsal lobe of the prostate among the N.C., BPH, and Fina groups (Fig. $3 \&$ Table 2).

p62 expression was primarily observed in epithelial cells (Fig. 4 \& Table 3). In the N.C. group, p62 expression was mostly confined to the epithelial cells of the anterior lobe, with almost no expression observed in other lobes. In contrast, cytoplasmic p62 expression was diffused expression in the epithelial cells in the prostate in the BPH group. In particular, the number of p62-positive cells increased in all lobes barring the anterior lobe compared to that in the N.C. group. p62 expression decreased significantly $(p<0.001)$ in the ventral lobe of the prostate in Fina group compared to that in the BPH group. p62 expression increased in the anterior lobe cells and reduced marginally in the dorsal and ventral lobe cells in the Fina group compared to that in the BPH group. In the Fina group, the ventral lobe cells showed partial expression of p62 compared to cells in the other lobes. 
Table 2. The intensity scoring of $1 \mathrm{~A} / 1 \mathrm{~B}$-light chain 3 in testosterone-induced benign prostate hyperplasia in rat

\begin{tabular}{lccc}
\hline & \multicolumn{3}{c}{ LC3 } \\
& N.C. & BPH & Fina \\
\hline Anterior lobe & ++ & + & + \\
Dorsal lobe & + & + & + \\
Lateral lobe & +++ & - & ++ \\
Ventral lobe & ++ & - & ++ \\
\hline
\end{tabular}

Testosterone-induced BPH rat models were administered finasteride $(10 \mathrm{mg} /$ $\mathrm{kg} \mathrm{P.O.)} \mathrm{for} 28$ days. The IHC test gives a score of 0 tor $3+$ that measures the intensity of LC3B- and p62-positive cells in the prostate tissue samples; one or little stained cells (0); weak, partial stained cells (1); intermediate, complete stained cells (2); strong, complete membrane stained cells (3).
Table 3. The intensity scoring of sequestosome 1in testosteroneinduced benign prostate hyperplasia in rat

\begin{tabular}{lccc}
\hline & & p62 & \\
& N.C. & BPH & Fina \\
\hline Anterior lobe & ++ & ++ & ++ \\
Dorsal lobe & - & ++ & + \\
Lateral lobe & - & ++ & + \\
Ventral lobe & - & ++ & - \\
\hline
\end{tabular}

Testosterone-induced BPH rat models were administered finasteride $(10 \mathrm{mg} /$ $\mathrm{kg}$ P.O.) for 28 days. The IHC test gives a score of 0 tor $3+$ that measures the intensity of LC3B- and p62-positive cells in the prostate tissue samples; one or little stained cells (0); weak, partial stained cells (1); intermediate, complete stained cells (2); strong, complete membrane stained cells (3).
A

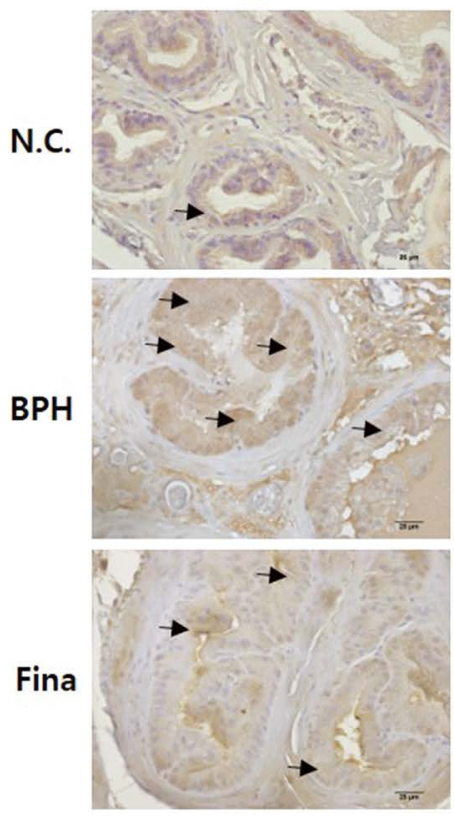

$\mathrm{DL}$

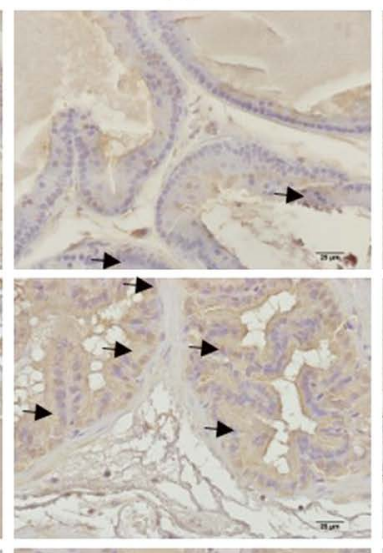

LL

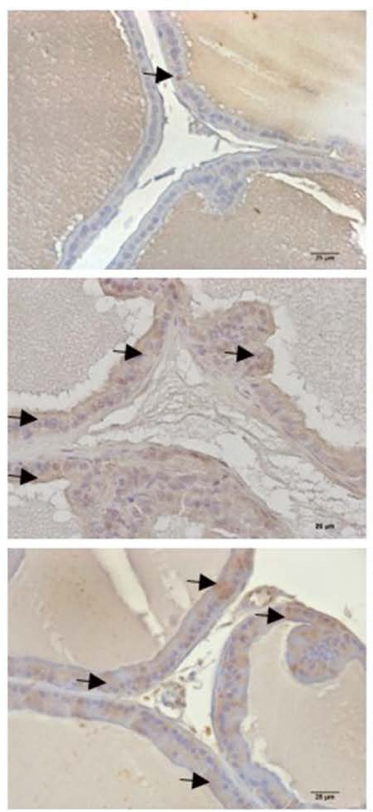

VL
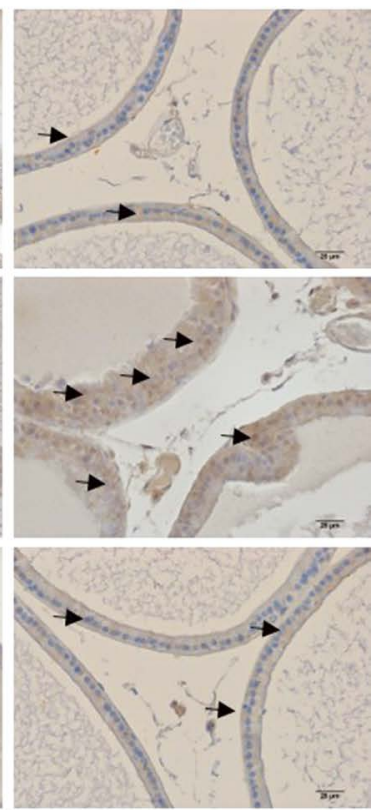

B

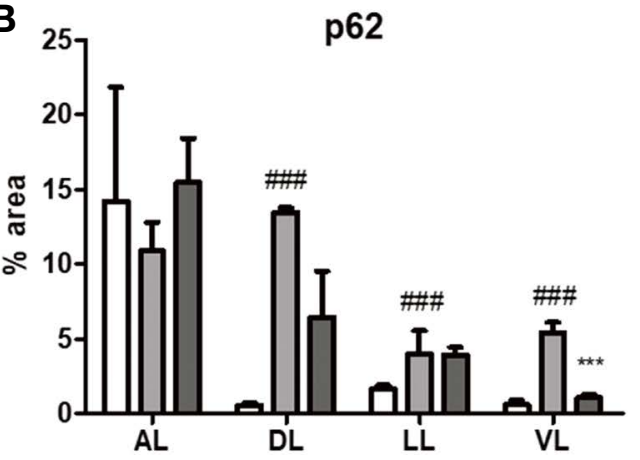

Fig. 4. Immunohistochemistry of p62 in a rat model of testosterone-induced BPH. Scale bar $=25 \mu \mathrm{m}$. Testosterone-induced BPH rat models were administered finasteride ( $10 \mathrm{mg} / \mathrm{kg}$ P.O.) for 28 days. A. Expression of the p62 (arrow) and examined by light microscopy with focus on AL, DL, LL, and VL. The\% area of positive p62(B) was expressed as mean \pm standard deviation, AL (anterior lobe), DL(dorsal lobe), LL (lateral lobe), and VL (ventral lobe), ${ }^{\# \# \# ~} p<0.001$, a significant difference in comparison with the N.C. group. $* * p<0.001$, significant difference in comparison with the BPH group. 


\section{DISCUSSION}

Anatomically, BPH diagnosis is established by enlarged size and increased weight of the prostate gland. Histologically, it is based on the proliferation of epithelial and connective tissues in the transition zone of the prostate lobes [30]. Although BPH pathogenesis is unclear, it has been associated with androgen expression $[1,12]$. Increased prostate volume is attributed to decreased apoptosis in the prostate rather than the prostate tissue proliferation [31,32]. Additionally, a recent study further reported that while cellular autophagy plays a compensatory role, it also provides a positive feedback to apoptosis in the process [31]. Autophagy, which maintains cellular homeostasis, is one of the cell death mechanisms. Blockade of $5 \alpha$-reductase type 2 action has been shown to increase autophagy [21]. LC3B, which is present on the inner membrane of the autophagosome, binds to p62 to form the autophagosome which is preferentially degraded during autophagy. Generally, autophagy is characterized by increased LC3B expression and decreased p62 expression, which collectively constitute the "autophagy flux" [19,33,34]. LC3B and p62 are degraded along with the autophagosome; therefore, these two factors should be considered in conjunction when studying autophagy [35].

Autophagy is suppressed in patients who suffer from severe prostatic inflammation with LUTS and BPH. These patients showed high expression of p62 and a low level of LC3B [25]. In the process of androgen-induced prostatic hyperplasia in castrated rats, androgen may be related to the phosphoinositide 3-kinase/protein kinase $\mathrm{B} /$ mechanistic target of rapamycin (PI3K/Akt/mTOR) pathway [35]. The prostate of the BPH exhibits decreased expression of LC3B, which showed the defective in autophagy flux [26]. In addition, testosterone up-regulates androgen-binding protein expression by p62 accumulation that is autophagy clearance inhibition [36]. However, the site for action of autophagy in the rat prostate lobe is yet to be identified. As the result of the Oh et al. study [26], our western blotting results also showed that the protein expression LC3B is decreased and p62 is increased in rat BPH prostate compared with normal prostate. To evaluate the localization of prostatic lobe BPH, the histological examination was focused on the expression and localization of LC3B and p62 in this study. In N.C. group, LC3B is expressed in the cytoplasm of epithelial cells. During BPH progression, LC3B expression decreased in the BPH group, particularly in the anterior, lateral, and ventral lobes of the prostate. However, Fina group showed that the number of LC3B-positive cells was increased especially in the lateral and ventral lobes of the prostate. In N.C. group, p62 was mostly expressed in the cytoplasm of epithelial cells in the anterior lobes of the prostate. p62 expression increased in the BPH group, particularly in the dorsal, lateral, and ventral lobes of the prostate. In the Fina group, p62 expression decreased to the levels similar to those observed in the N.C. group.

Both LC3B expression and p62 expression were higher in the anterior lobe than other areas. In addition, in the Fina group, p62 expression was higher than in the BPH group. Ultrastructurally, the cells of anterior lobe have a dilated endoplasmic reticulum recognized as dilated endoplasmic reticulum (ER) lumen, which indicates an increase in ER stress [37]. ER stress is one of the inducers of autophagy, and it is implied that the expression of LC3B was higher in the anterior lobe than in other areas. p62 is a autophagy flux marker and is accumulated when autophagy is inhibited [38]. These results suggested that the formation of autophagosome occurs a lot in the anterior lobe, however, the autophagosome clearance does not occur completely.

There was no significant difference in the dorsal lobe LC3B expression among the N.C., BPH, and Fina groups. However, p62 expression was substantially higher in the $\mathrm{BPH}$ group than in the N.C. group. p62 expression reduced in the Fina group; however, the extent of reduction was no comparable to that in the N.C. group. These results indicate that autophagy was reduced in the BPH and Fina groups, but not to the same degree as that in the N.C. group.

In the lateral lobe of the prostate, the number of LC3Bpositive cells was higher than that in the dorsal and ventral lobes in the N.C. group. However, LC3B expression was almost undetectable in the BPH group. LC3B expression in the Fina group was not substantially higher than that in N.C. group. p62 expression in the BPH and Fina groups increased compared to that in the N.C. group. Therefore, autophagy was reduced in the lateral lobe during BPH and finasteride treatment.

In the ventral lobe of the prostate, LC3B expression was lower in the BPH group, whereas it was higher in the Fina group (the levels were similar to those observed in the N.C. group). On the other hand, p62 expression increased in the $\mathrm{BPH}$ group, whereas it was lower in the Fina group similar to those observed in the N.C. group. These Wesults indicated that the autophagy flux in the ventral lobe reduced in $\mathrm{BPH}$, 
whereas it increased in response to finasteride treatment during $\mathrm{BPH}$. Because ventral lobe of prostate has a greater androgen responsiveness than the other lobes, the spontaneously occurring benign proliferative lesions are more common in the ventral prostate [39]. Androgen may be related o the PI3K/Akt/mTOR signaling pathway inhibiting autophagy flux [35]. Under finasteride treatment, the reduction of androgen activity induced autophagy. Histologically, the lateral lobe more closely resembles the ventral lobe [39] and both of them are dependent of DHT [40]. Under BPH, autophagy was reduced with LC3B increase and p62 decrease in lateral and ventral lobe. However, the mean ratio of DHT to testosterone levels in lateral lobe was six times greater than ventral lobe [40]. This difference affect to the effectiveness of finasteride on autophagy flux in lateral lobe.

Although the incidence of autophagy was reduced in the BPH group, cell proliferation was increased during $\mathrm{BPH}$. It is known to the unbalance between apoptosis and proliferation cause the $\mathrm{BPH}$. Androgen signaling drove cell progression by decreasing p27kip1 in prostate [41]. However, finasteride treatment induced LC3B expression and reduced p62 expression, suggesting normal autophagy flux. The LC3B and p62 expression pattern was consistent with the western blotting results. In the pathogenesis and treatment of BPH, studies have been conducted on the relationship between androgen signaling and autophagy process $[21,42,43]$. These findings collectively indicated that altered LC3B and p62 expression in the ventral lobe in the Fina group was associated with localized autophagy during $\mathrm{BPH}$. The rat prostate is characterized by histological and functional heterogeneity between the lobes [10]. In the BPH group, LC3B expression decreased and p62 expression was increased in all areas with cell proliferation. However, finasteride treatment induced autophagy in the epithelial cells of the dorsal, lateral, and ventral lobes, with the ventral lobe showing most prominent autophagy flux.

The IHC results showed that glandular cells are involved in autophagy, and resultantly, in BPH prevention. Despite the fact that autophagy was only evaluated by measuring the expression of key autophagy markers such as LC3B and p62, which is a limitation of this study, the markers can be used to determine the specific site of autophagy.

Autophagy flux was decreased in patients and animal models of BPH, whereas it is known to increase under treatment with androgen receptor inhibitor. However, the precise local site of autophagy has not been elucidated in $\mathrm{BPH}$ progression and treatment $[42,44,45]$. Our data collectively indicated that the ventral lobe, which constitutes the major part of prostate, is a prominent site of autophagy during $\mathrm{BPH}$. It could be seen that the ventral lobe is correspond to transitional zone during the pathogenesis and treatment of $\mathrm{BPH}$. Based on our finding, we suggest that autophagy is a critical process in BPH. In particular, the ventral lobe of the rat prostate could be a potential target site for evaluating the therapeutic effects of BPH treatment in animal models.

\section{REFERENCES}

1. Roehrborn CG. Benign prostatic hyperplasia: an overview. Rev Urol. 2005;7(Suppl 9):S3-S14.

2. Djavan B. Lower urinary tract symptoms/benign prostatic hyperplasia: fast control of the patient's quality of life. Urology. 2003;62(3 Suppl 1):6-14.

3. Scolnik MD, Servadio C, Abramovici A. Comparative study of experimentally induced benign and atypical hyperplasia in the ventral prostate of different rat strains. J Androl. 1994; 15:287-97.

4. Shirai T, Takahashi S, Cui L, Futakuchi M, Kato K, Tamano S, et al. Experimental prostate carcinogenesis - rodent models. Mutat Res-Rev Mutat. 2000;462:219-26.

5. Shirai T. Significance of chemoprevention for prostate cancer development: Experimental in vivo approaches to chemoprevention. Pathol Int. 2008;58:1-16.

6. Nascimento-Goncalves E, Faustino-Rocha AI, Seixas F, Ginja M, Colaco B, Ferreira R, et al. Modelling human prostate cancer: Rat models. Life Sci. 2018;203:210-24.

7. Bosland MC. Chemical and hormonal induction of prostate cancer in animal models. Urol Oncol. 1996;2:103-10.

8. Aumuller G, Enderle-Schmitt U, Seitz J, Muntzing J, Chandler JA. Ultrastructure and immunohistochemistry of the lateral prostate in aged rats. Prostate. 1987;10:245-56.

9. Lundgren R, Holmquist B, Hesselvik M, Muntzing J. Treatment of prostatitis in the rat. Prostate. 1984;5:277-84.

10. Hayashi N, Sugimura Y, Kawamura J, Donjacour AA, Cunha GR. Morphological and functional heterogeneity in the rat prostatic gland. Biol Reprod. 1991;45:308-21.

11. Isaacs JT. Development and characteristics of the available animal model systems for the study of prostatic cancer. Prog Clin Biol Res. 1987;239:513-76.

12. Bruchovsky N, Lesser B, Van Doorn E, Craven S. Hormonal effects on cell proliferation in rat prostate. Vitam Horm. 1975;33:61-102.

13. Kroemer G, Levine B. Autophagic cell death: the story of a misnomer. Nat Rev Mol Cell Biol. 2008;9:1004-10. 
14. Farrow JM, Yang JC, Evans CP. Autophagy as a modulator and target in prostate cancer. Nat Rev Urol. 2014;11:508-16.

15. Levine B, Kroemer G. Autophagy in the pathogenesis of disease. Cell. 2008;132:27-42.

16. Mizushima N, Yoshimori T, Levine B. Methods in mammalian autophagy research. Cell. 2010;140:313-26.

17. Kabeya Y, Mizushima N, Ueno T, Yamamoto A, Kirisako T, Noda T, et al. LC3, a mammalian homologue of yeast Apg8p, is localized in autophagosome membranes after processing. EMBO J. 2000;19:5720-8.

18. Komatsu M, Waguri S, Koike M, Sou YS, Ueno T, Hara T, et al. Homeostatic levels of p62 control cytoplasmic inclusion body formation in autophagy-deficient mice. Cell. 2007; 131:1149-63.

19. Kliosnky D. Guidelines for the Use and Interpretation of Assays for Monitoring Autophagy (3rd edition) (vol 12,pg 1, 2015). Autophagy. 2016;12:445-544.

20. Komatsu M, Ichimura Y. Physiological significance of selective degradation of p62 by autophagy. Febs Letters. 2010; 584:1374-8.

21. Li M, Yang X, Wang H, Xu E, Xi Z. Inhibition of androgen induces autophagy in benign prostate epithelial cells. Int J Urol. 2014;21:195-9.

22. Liu C, Xu P, Chen D, Fan X, Xu Y, Li M, et al. Roles of autophagy-related genes Beclin-1 and LC3 in the development and progression of prostate cancer and benign prostatic hyperplasia. Biomed Rep. 2013;1:855-60.

23. Zhang N, Ji N, Jiang WM, Li ZY, Wang M, Wen JM, et al. Hypoxia-induced autophagy promotes human prostate stromal cells survival and ER-stress. Biochem Biophys Res Commun. 2015;464:1107-12.

24. Ruan Y, Jiang CY, Wang XH, Jiang Q, Han BM, Sun XW, et al. Clinical relevance and implications of autophagy-related proteins in benign prostatic hyperplasia. Int J Clin Exp Patho. 2017;10:4705-18.

25. De Nunzio C, Giglio S, Stoppacciaro A, Gacci M, Cirombella

$\mathrm{R}$, Luciani E, et al. Autophagy deactivation is associated with severe prostatic inflammation in patients with lower urinary tract symptoms and benign prostatic hyperplasia. Oncotarget. 2017;8:50904-10.

26. Oh SH, Lee DW, Choi YB, Lee YH, Ju JS. Measurement of autophagy flux in benign prostatic hyperplasia in vitro. Prostate Int. 2020;8:70-7.

27. Hong GL, Park SR, Jung DY, Karunasagara S, Lee KP, Koh EJ, et al. The therapeutic effects of Stauntonia hexaphylla in benign prostate hyperplasia are mediated by the regulation of androgen receptors and 5 alpha-reductase type 2. J Ethnopharmacol. 2020;250.

28. Karunasagara S, Hong GL, Jung DY, Kim KH, Cho K, Jung JY. Protective effects of combination of Stauntonia hexa- phylla and Cornus officinalis on testosterone-induced benign prostatic hyperplasia through inhibition of 5alpha- reductase type 2 and induced cell apoptosis. PLoS One. 2020;15: e0236879.

29. McCullough D. The effect of finasteride in men with benign prostatic hyperplasia - Editorial comment. J Urology. 2002; 167:1185-91.

30. Cornu JN, Oelke M, Parsons KF. Benign prostatic hyperplasia and lower urinary tract symptoms. N Engl J Med. 2012;367:1668; author reply-9.

31. Li M, Jiang X, Liu D, Na Y, Gao GF, Xi Z. Autophagy protects $\mathrm{LNCaP}$ cells under androgen deprivation conditions. Autophagy. 2008;4:54-60.

32. Xie W, Wong YC, Tsao SW. Correlation of increased apoptosis and proliferation with development of prostatic intraepithelial neoplasia (PIN) in ventral prostate of the Noble rat. Prostate. 2000;44:31-9.

33. Deretic V, Klionsky DJ. Autophagy and inflammation: A special review issue. Autophagy. 2018;14:179-80.

34. Huang WP, Klionsky DJ. Autophagy in yeast: a review of the molecular machinery. Cell Struct Funct. 2002;27:409-20.

35. Liu RF, Fu G, Li J, Yang YF, Wang XG, Bai PD, et al. Roles of autophagy in androgen-induced benign prostatic hyperplasia in castrated rats. Exp Ther Med. 2018;15:2703-10.

36. Ma Y, Yang HZ, Xu LM, Huang YR, Dai HL, Kang XN. Testosterone regulates the autophagic clearance of androgen binding protein in rat Sertoli cells. Sci Rep-Uk. 2015;5.

37. Ding WX, Ni HM, Gao WT, Hou YF, Melan MA, Chen XY, et al. Differential effects of endoplasmic reticulum stressinduced autophagy on cell survival. J Biol Chem. 2007;282: 4702-10.

38. Bjorkoy G, Lamark T, Pankiv S, Overvatn A, Brech A, Johansen T. Monitoring autophagic degradation of p62/ SQSTM1. Methods Enzymol. 2009;452:181-97.

39. Whitney KM. Boorman's Pathology of the Rat Second Edition) ed: Elesevier; 2018. p. 8.

40. Prahalada S, Rhodes L, Grossman SJ, Heggan D, Keenan KP, Cukierski MA, et al. Morphological and hormonal changes in the ventral and dorsolateral prostatic lobes of rats treated with finasteride, a 5-alpha reductase inhibitor. Prostate. 1998;35:157-64

41. Simone de Brot, Mongan NP. Precision Molecular Pathology of Prostate Cancer: Springer; 2017. p. 16.

42. Ding M, Jiang CY, Zhang Y, Zhao J, Han BM, Xia SJ. SIRT7 depletion inhibits cell proliferation and androgen-induced autophagy by suppressing the AR signaling in prostate cancer. J Exp Clin Cancer Res. 2020;39:28.

43. Liu RF, Fu G, Li J, Yang YF, Wang XG, Bai PD, et al. Roles of autophagy in androgen-induced benign prostatic hyperplasia in castrated rats. Exp Ther Med. 2018;15:2703-10. 
44. Yang BY, Jiang CY, Dai CY, Zhao RZ, Wang XJ, Zhu YP, et al. 5-ARI induces autophagy of prostate epithelial cells through suppressing IGF-1 expression in prostate fibroblasts. Cell Prolif. 2019;52:e12590.
45. Jiang CY, Yang BY, Zhao S, Shao SH, Bei XY, Shi F, et al. Deregulation of ATG9A by impaired AR signaling induces autophagy in prostate stromal fibroblasts and promotes BPH progression. Cell Death Dis. 2018;9:431. 